\title{
Effects of Communication Impairments on Quorum Sensing
}

\author{
Benjamin E. Beckmann, Philip K. McKinley and David B. Knoester \\ Department of Computer Science and Engineering \\ Michigan State University, East Lansing, Michigan \\ Email: $\{$ beckma24,mckinley,dk\}@ cse.msu.edu
}

Motivations. Observations of behaviors in natural organisms provide useful insight into the complexity of selforganizing systems and the building blocks that underpin them. A behavior that is important to both biological and computational systems is quorum sensing (QS) [1], where sufficient group density triggers a collective group action. Quorum-sensing bacteria, for example, continually secrete and detect molecules called autoinducers (AIs) [2]. Under low-density conditions, AI molecules diffuse through the environment and go undetected. As the number of bacterial cells increases, so does the level of AI, eventually reaching a threshold that triggers a change in gene expression. Studies have shown that bacteria use quorum sensing in a wide variety of functions. In pathogenic bacteria, for instance, quorum sensing enables organisms to coordinate attacks and express virulence factors in sufficient quantities to overwhelm the host's immune response [3].

Many distributed computing systems also rely on QS to operate correctly. Most notably, algorithms for consensus, where members of a group agree on a particular course of action, represent a special-case of QS. Consensus algorithms support distributed lock management, consistency of replicated components, and distributed control systems. Sensor networks use QS to perform clustering, where nodes are divided into groups in order to perform data aggregation [4]. QS has also been proposed to coordinate behavior among multiple instances of a computer worm [5]. For example, Vogt et al. [5] discuss methods by which a worm could spread to an intended number of hosts and stop once a quorum is reached, lowering its profile.

Methods to prevent or disrupt QS, referred to as quorum quenching, can serve multiple purposes. For example, quorum quenching can be used to test the robustness of a distributed system, revealing weaknesses in the design before the system is deployed. In addition, quorum quenching has been proposed as a means to reduce the virulence of pathogenic bacteria [6], [7]. An important concern, however, is whether target systems, either natural or computational, might develop resistance to quorum quenching methods. In this work we investigate the evolution of resistance to quorum quenching techniques, in an attempt to help predict outcomes and discover treatments in both biological and computational domains.
Digital Evolution. To facilitate this research we employ the Avida digital evolution platform [8]. Avida is a well established computational tool used in evolutionary biology [9] and more recently in distributed systems research [10]. In Avida, digital organisms compete for space within a fixed-size two-dimensional collection of cells. Each cell can contain at most one organism, which comprises a circular list of instructions (its genome) and a virtual CPU that executes those instructions. Avida organisms are selfreplicating, that is, their genomes must contain instructions to create offspring. An Avida population starts with an ancestral organism capable only of replication. As replications occur, instruction-level mutations produce variation within the population.

Avida organisms communicate by sending messages to one another. We extended Avida with an interrupt model similar to the execution model of TinyOS [11]. In this model an organism's main execution thread can be interrupted by an event, such as receiving a message. To enable the evolution of an interrupt handler, we introduced two instructions that denote the beginning (msg-handler) and end (endhandler) of an interrupt handler. We emphasize that these instructions have simply been added to the set of instructions available for mutation into an organism's genome. Whether they are used is solely a result of natural selection.

A population of organisms can be subdivided into multiple sub-populations, called demes. All demes have identical environments and initial configurations, and an organism can interact only with other organisms in its deme. Subdividing the population this way facilitates the detection of and selection for group-level behaviors.

Quorum Quenching Experiments. Medical treatments that disrupt quorum sensing by introducing disabled mutants, specifically mutants incapable of sending (signalnegative) or receiving (signal-blind) AI molecules, have shown promise [7]. Effectively, the mutants act as cheaters, exploiting the cooperative production of virulence factors, but not fully participating in the underlying QS behavior. Rumbaugh et al. [7] showed that receive-impaired mutants reduced host mortality rate more than send-impaired mutants, indicating that different impairments disrupt QS activity to varying degrees.

In a prior study [12], we demonstrated that digital or- 
ganisms can evolve to perform QS as follows. Before a quorum has been reached, the organisms send a few messages and self-replicate. However, as a population of organisms increases in size, so does the number of messages sent. At quorum these messages cause the organisms to become and remain interrupted. To evaluate the effectivess of quorum quenching, we observe how digital organisms fare in environments where offspring are probabilistically impaired at birth so they cannot send or receive messages, respectively.

In this study, we extend runs from [12] from 2,500 deme generations to 5,000 and observed 16 runs that evolved QS behavior. We then tested the robustness of the most abundant (dominant) genomes to the introduction of impaired mutants. To perform tests similar to those done with mice in [7], we inject a single seed organism into all demes and measure the fraction of demes that contain "living" organisms after one competition period, its survival rate.

Figure 1 shows a sample of the results: the mean fraction of demes surviving at the conclusion of a competition period, interpolated over the range of 0 to 10 mutants, sampled at mutant introduction rates of $0,1,2,5$, and 10 per 100 births. The introduction of send-impaired mutants had minimal effect on the number of demes that survived for a single competition period. Only 549 out of 6400 demes did not survive at the highest introduction rate of send impaired mutants $(10 \%)$ and the lowest introduction rate of receive impaired mutants (0\%). However, demes that were subjected to receive-impaired mutants exhibited an increase in deme mortality corresponding to an increase in mutant births. Based on these data, a logical quorum quenching treatment is to introduce receive-impaired mutants into the demes. At a introduction rate of $10 \%$, such mutants killed approximately $88.4 \%$ of all demes tested.

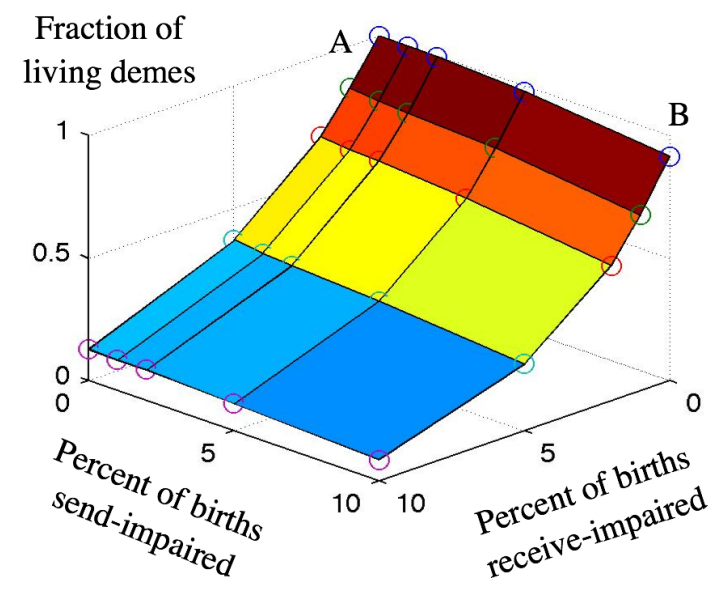

Figure 1. Fraction of living demes after a competition period.
Summary. Many natural and artificial systems utilize QS to perform critical tasks, from self-preservation in bacteria to clustering in sensor networks. The disruption of these systems can serve many purposes, from treating diseasecausing bacteria to disabling computer worms. We have shown that digital evolution can produce solutions that are resistant to communication impairments. We demonstrated that QS digital organisms exhibit a resistance to communication impairments. Building on this work, we intend to test the effects of other types of therapies to prevent and promote QS.

Acknowledgments. This work was supported by NSF Grants CCF-0750787, CNS-0751155, CCF-0820220, by U.S. Army Grant W911NF-08-1-0495, and by a Quality Fund Grant from Michigan State University.

\section{REFERENCES}

[1] C. M. Waters and B. L. Bassler, "Quorum sensing: Cell-tocell communication in bacteria," Annual Review of Cell and Developmental Biology, vol. 21, pp. 319-346, 2005.

[2] K. H. Nealson, T. Platt, and J. W. Hastings, "Cellular control of the synthesis and activity of the bacterial luminescent system," J Bacteriol., vol. 104, no. 1, pp. 313-322, 1970.

[3] T. R. de Kievit and B. H. Iglewski, "Bacterial quorum sensing in pathogenic relationships," Infect. Immun., vol. 68, no. 9, pp. 4839-4849, September 2000. [Online]. Available: http://dx.doi.org/10.1128/IAI.68.9.4839-4849.2000

[4] G. Chockler, S. Gilbert, and B. Patt-Shamir, "Communication-efficient probabilistic quorum systems for sensor networks," in Proceedings of the 4th Annual IEEE International Conference on Pervasive Computing and Communications Workshops. Los Alamitos, CA, USA: IEEE Computer Society, March 2006, pp. 111-117.

[5] R. Vogt, J. Aycock, and M. J. Jacobson, "Quorum sensing and self-stopping worms," in Proceedings of the 2007 ACM Workshop on Recurring Malcode. New York, NY, USA: ACM, 2007, pp. 16-22.

[6] K. B. Xavier and B. L. Bassler, "Interference with AI-2mediated bacterial cell-cell communication," Nature, no. 437, pp. 750-753, 2005.

[7] K. P. Rumbaugh, S. P. Diggle, C. M. Watters, A. RossGillespie, A. S. Griffin, and S. A. West, "Quorum sensing and the social evolution of bacterial virulence," Current Biology, vol. 19, no. 4, pp. 341-345, January 2009.

[8] C. Ofria and C. O. Wilke, "Avida: A software platform for research in computational evolutionary biology," Artificial Life, vol. 10, pp. 191-229, March 2004. [Online]. Available: http://alife.org

[9] R. E. Lenski, C. Ofria, R. T. Pennock, and C. Adami, "The evolutionary origin of complex features," Nature, vol. 423, pp. 139-144, 2003.

[10] P. McKinley, B. Cheng, C. Ofria, D. Knoester, B. Beckmann, and H. Goldsby, "Harnessing digital evolution," IEEE Computer, vol. 41, no. 1, pp. 54-63, January 2008.

[11] J. L. Hill, "System architecture for wireless sensor networks," Ph.D. dissertation, University of California at Berkeley, 2003.

[12] B. E. Beckmann and P. K. McKinley, "Evolving quorum sensing in digital organisms," in Proceedings of the 11th Annual Conference on Genetic and Evolutionary Computation, 2009, in press. 